\title{
An Elementary Proof of the Theorems of Cauchy and Mayer
}

\author{
By A. J. Macintyre, University of Sheffield.
}

Communicated by R. WiLson.

(Received 19th November, 1934. Read 7th December, 1934.)

\section{Introduction.}

Attention has recently been drawn ${ }^{1}$ to the obscurity of the usual presentations of Mayer's method of solution of the total differential equation

$$
d z=P(x, y, z) d x+Q(x, y, z) d y .
$$

This method has the practical advantage that only a single integration is required, but its theoretical discussion is usually based on the validity of some other method of solution. ${ }^{2}$ Mayer's method gives a result even when the equation ( 1 ) is not integrable, but this cannot of course be a solution. An examination of the conditions under which the result is actually an integral of equation (1) leads to a proof of the existence theorem for (1) which is related to Mayer's method of solution in a natural way, and which moreover appears to be novel and of value in the presentation of the subject.

The problem of the conditions under which the expression

$$
P(x, y) d x+Q(x, y) d y
$$

is the total differential of a function of $x$ and $y$ can also be discussed with advantage from the same point of view. The extension of the calculus to complex variables is dependent on this problem, and sufficient conditions for Cauchy's fundamental theorem are here obtained. These conditions are the same as those which arise in the proof of Cauchy's theorem by means of Green's theorem, but the method may well be considered to be simpler and, in addition, it affords a suitable approach to this subject for the many students who are interested only in its most elementary aspect.

In order to facilitate the reading, the simpler argument leading to Cauchy's theorem is first given and the discussion of Mayer's method then follows. 
2. Total differentials and Cauchy's Theorem.

Cauchy's theorem may be considered as the extension to complex variables of the fundamental theorem of calculus concerning the relation between differentiation and integration. A very natural but apparently new method of discussing this question is to follow closely the method of the real variable which considers a certain definite integral as a function of one of its limits.

Starting then from the differential expression

$$
P(x, y) d x+Q(x, y) d y,
$$

we consider its integral taken along a straight line from a fixed point, say the origin, to the point $(x, y)$. That is to say we consider the function ${ }^{3}$

$$
F(x, y)=\int_{0}^{1}\{x P(x t, y t)+y Q(x t, y t)\} d t .
$$

It is evident that if (2) is a total differential at all, then it is the total differential of the function (3). It is easy to investigate this question by calculating the partial derivatives of (3). For example,

$$
\begin{aligned}
\frac{\partial}{\partial x} F(x, y) & =\int_{0}^{1}\left\{P(x t, y t)+x t \frac{\partial P(x t, y t)}{\partial(x t)}+y t \frac{\partial Q(x t, y t)}{\partial(x t)}\right\} d t \\
& =\int_{0}^{1}\left[\frac{\partial}{\partial t}\{t P(x t, y t)\}+y t\left\{\frac{\partial Q(x t, y t)}{\partial(x t)}-\frac{\partial P(x t, y t)}{\partial(y t)}\right\}\right] d t \\
& =P(x, y)+\int_{0}^{1} y t\left\{\frac{\partial Q(x t, y t)}{\partial(x t)}-\frac{\partial P(x t, y t)}{\partial(y t)}\right\} d t .
\end{aligned}
$$

Sufficient conditions for the validity of these calculations are that $P(x, y), Q(x, y)$ and their partial derivatives of the first order are continuous. ${ }^{4}$

If further

identically, then

$$
\frac{\partial}{\partial y} P(x, y)=\frac{\partial}{\partial x} Q(x, y)
$$

Similarly under the same conditions

$$
\frac{\partial}{\partial y} F(x, y)=Q(x, y) \text {. }
$$


Thus are established the well known conditions under which (2) is a total differential, for if $F(x, y)$ has the continuous derivatives $P(x, y)$ and $Q(x, y)$, then $(2)$ is its total differential.

If $f(z)=u+i v$ is a function of the complex variable $z=x+i y$ then from (5), (6) and (7),

$$
f(z) d z=f(z) d x+i f(z) d y
$$

will be a total differential if

$$
\frac{\partial f(z)}{\partial y}=i \frac{\partial f(z)}{\partial x},
$$

and on equating real and imaginary parts in this equation we have the Cauchy-Riemann equations

$$
\frac{\partial u}{\partial x}=\frac{\partial v}{\partial y}, \quad \frac{\partial u}{\partial y}=-\frac{\partial v}{\partial x} .
$$

For the validity of this argument we require ${ }^{5}$ the continuity of the partial derivatives of $u$ and $v$.

3. The equation $d z=P(x, y, z) d x+Q(x, y, z) d y$.

In this article we discuss Mayer's method of solution of the equation (1) in a way that establishes the usual existence theorem for the equation and requires reference only to the necessary parts of the theory of ordinary differential equations.

We recall that condition (5) which expresses that $\frac{\partial^{2} z}{\partial x \partial y}=\frac{\partial^{2} z}{\partial y \partial x}$ must now be replaced by

or

$$
\frac{\partial Q}{\partial x}+\frac{\partial Q}{\partial z} \frac{\partial z}{\partial x}=\frac{\partial P}{\partial y}+\frac{\partial P}{\partial z} \frac{\partial z}{\partial y}
$$$$
P Q_{z}-Q P_{z}+Q_{x}-P_{y}=0,
$$

and this relation is generally termed the condition of integrability of equation (1).

If $z$ is a function of $x$ and $y$ which satisfies equation (1) and we replace $x$ and $y$ by $x t$ and $y t$ and take $t$ as a variable, $x$ and $y$ being fixed, then $z$ evidently satisfies

$$
d z=\{x P(x t, y t, z)+y Q(x t, y t, z)\} d t .
$$

This is an ordinary differential equation of the first order and will have a solution reducing to $z_{0}$ for $t=0$ defined for the range $0 \leqq t \leqq 1$, for $x$ and $y$ in some neighbourhood of the origin, provided 
that $P$ and $Q$ are continuous functions of their arguments and satisfy a Lipschitz condition with respect to $z$ for $(x, y, z)$ in some neighbourhood $^{6}$ of $\left(0,0, z_{0}\right)$.

This solution depends on the parameters of $x, y, z_{0}$ and will be denoted by

$$
z=F\left(x, y, z_{0}, t\right)
$$

For $t=1$ we write

$$
z=F\left(x, y, z_{0}, 1\right)=\psi\left(x, y, z_{0}\right) \text {. }
$$

The whole question turns on establishing the relations

$$
\frac{\partial}{\partial x} \psi\left(x, y, z_{0}\right)=P(x, y, z), \quad \frac{\partial}{\partial y} \psi\left(x, y, z_{0}\right)=Q(x, y, z) .
$$

Since $F\left(x, y, z_{0}, t\right)$ satisfies (8) we have formally

$$
\begin{aligned}
\frac{\partial^{2} F}{\partial t \partial x}=\frac{\partial^{2} F}{\partial x \partial t}=\frac{\partial}{\partial x} & \{x P(x t, y t, z)+y Q(x t, y t, z)\} \\
=P(x t, y t, z) & +x t \frac{\partial P(x t, y t, z)}{\partial(x t)}+x \frac{\partial P(x t, y t, z)}{\partial z} \lambda\left(x, y, z_{0}, t\right) \\
& +y t \frac{\partial Q(x t, y t, z)}{\partial(x t)}+y \frac{\partial Q(x t, y t, z)}{\partial z} \lambda\left(x, y, z_{0}, t\right),
\end{aligned}
$$

where we write

$$
\lambda\left(x, y, z_{0}, t\right) \equiv \frac{\partial}{\partial x} F\left(x, y, z_{0}, t\right) \equiv \frac{\partial z}{\partial x}
$$

from (9). Hence from (12) and (8),

$$
\begin{array}{r}
\frac{\partial}{\partial t} \lambda(x, y, z, t)=\frac{\partial}{\partial t}\{t P(x t, y t, z)\}+y t\left\{\frac{\partial Q(x t, y t, z)}{\partial(x t)}-\frac{\partial P(x t, y t, z)}{\partial(y t)}\right\} \\
+\lambda(x, y, z, t)\left\{x \frac{\partial P(x t, y t, z)}{\partial z}+y \frac{\partial Q(x t, y t, z)}{\partial z}\right\} \\
-t \frac{\partial P(x t, y t, z)}{\partial z}\{x P(x t, y t, z)+y Q(z t, y t, z)\},
\end{array}
$$

giving

$$
\frac{\partial}{\partial t}(\lambda-t P)=(\lambda-t P)\left(x P_{z}+y Q_{z}\right)+y t\left(P Q_{z}-Q P_{z}+\frac{\partial Q}{\partial(x t)}-\frac{\partial P}{\partial(y t)}\right) .
$$

Hence if the condition of integrability

$P(x, y, z) Q_{z}(x, y, z)-Q(x, y, z) P_{z}(x, y, z)+Q_{x}(x, y, z)-P_{y}(x, y, z)=0$ is satisfied, then $\lambda\left(x, y, z_{0}, t\right)-t P(x t, y t, z)$ satisfies the differential equation

$$
\frac{\partial}{\partial t}(\lambda-t P)=(\lambda-t P)\left(x P_{z}+y Q_{z}\right) .
$$


Now when $t=0, F\left(x, y, z_{0}, t\right)=z_{0}$ and is independent of $x$ and $y$, so that from (12) $\lambda\left(x, y, z_{0}, 0\right)=0$, and hence $\lambda\left(x, y, z_{0}, t\right)-t P(x t, y t, z)$ is identically zero since there is only one solution of (15) with the required properties, ${ }^{8}$ and in particular from (10) and (13),

$$
\frac{\partial}{\partial x} \psi\left(x, y, z_{0}\right)=\lambda\left(x, y, z_{0}, 1\right)=P(x, y, z) .
$$

A similar argument will show that under the same conditions

$$
\frac{\partial}{\partial y} \psi\left(x, y, z_{0}\right)=Q(x, y, z) .
$$

Hence $z=\psi\left(x, y, z_{0}\right)$ is a solution of (2) and is the only solution of (2) which reduces to $z_{0}$ when $x$ and $y$ vanish.

We have thus proved the existence theorem:

THeorem. If $P(x, y, z)$ and $Q(x, y, z)$ are continuous and have continuous partial derivatives of the first order, and if

$$
P Q_{z}-Q P_{z}+Q_{x}-P_{y}=0
$$

in some neighbourhood of $\left(0,0, z_{0}\right)$, then the total differential equation

$$
d z=P(x, y, z) d x+Q(x, y, z) d y
$$

has one and only one solution

for which

$$
z=\psi(x, y)
$$

$$
\psi(0,0)=z_{0}
$$

defined in some neighbourhood ${ }^{9}$ of $(0,0)$.

\section{REFERENCES.}

1. M. L. Cartwright, Math. Gazette, XVIII (1934), 105-107.

2. Of. Goursat-Hedrick, Mathematical Analysis, Vol. II, part II (1917), p. 229, § 79.

3. This argument is strongly suggested by the concluding remarks of $\$ 152$, p. 318 in Goursat-Hedrick, A Course of Mathematical Analysis, Vol. I (1904), but does not seem to have been pursued. The remark is not to be found in French editions of Goursat's Cours d'Analyse.

4. Goursat-Hedrick, Vol. I, §97, pp. 192-3 (1904).

5. Cauchy, Euvres (1), 10, 70-74, uses Green's Theorem with the same conditions. E. Goursat, Trans. Amer. Math. Soc., 1 (1900), 14, and subsequent writers have shown that continuity is not an independent assumption. 
6. Goursat-Hedrick II, II, pp. 61-64. If $P(x, y, z)$ and $Q(x, y, z)$ are continuous and bounded by $M$ for $|x|<a,|y|<b$ and $|z|<c$, and satisfy a Lipschitz condition with respect to $z$, then the function

$$
f(z, t)=x P(x t, y t, z)+y Q(x t, y t, z)
$$

is continuous for these values of $x, y, z$, and for $|t| \leq 1$, and is bounded by $M(|x|+|y|) . f(z, t)$ also satisfies the Lipschitz condition with respect to $z$. The solution of the differential equation

$$
\frac{d z}{d t}=f(z, t)
$$

which reduces to $z_{0}$ for $t=t_{0}$ hence exists if $|t| \leq 1$ and $|t| \leq \frac{c}{M(|x|+|y|)}$, hence certainly for $|t| \leq 1$ if $|x|$ and $|y|$ are both less than $\frac{M}{2 c}$.

7. That $F\left(x, y, z_{0}, t\right)$ has continuous partial derivatives and that the following formal transformations are valid follows from the theory of ordinary differential equations involving parameters. See de la Vallée Poussin, Cours d'Analyse, 5th ed. (1925), Vol. II, Ch. 5, \$5, 133 ${ }^{\circ}$ pp. 147-149, and in particular p. $148,2^{\circ}$. The conditions required are that $P(x, y, z)$ and $Q(x, y, z)$ have continuous derivatives of the first order.

8. Or we may observe that the general solution of (15) is

$$
(\lambda-t P)=c \exp \left\{\int_{0}\left(x P_{z}+y Q_{z}\right) d t\right\}
$$

where $c$ is an arbitrary constant and $\lambda-t P$ must vanish identically or not at all.

9. The Lipschitz condition required for the solution of equation (8) (see Note 6 above) is satisfied on account of the conditions that $P_{z}$ and $Q_{z}$ are continuous. 